Revue d'histoire de l'Amérique française

\#A REVUE D.HISTOIRE DE L'AMÉRIQUE FRANÇAISE

\title{
Le congrès des métiers et du travail et la guerre civile espagnole
}

\section{Richard Martin}

Volume 33, numéro 4, mars 1980

URI : https://id.erudit.org/iderudit/303813ar

DOI : https://doi.org/10.7202/303813ar

Aller au sommaire du numéro

Éditeur(s)

Institut d'histoire de l'Amérique française

ISSN

0035-2357 (imprimé)

1492-1383 (numérique)

Découvrir la revue

Citer cette note

Martin, R. (1980). Le congrès des métiers et du travail et la guerre civile espagnole. Revue d'histoire de l'Amérique française, 33(4), 575-581.

https://doi.org/10.7202/303813ar d'utilisation que vous pouvez consulter en ligne.

https://apropos.erudit.org/fr/usagers/politique-dutilisation/ 
NOTE DE RECHERCHE

\title{
- I - \\ LE CONGRÈS DES MÉTIERS ET DU TRAVAIL ET LA GUERRE CIVILE ESPAGNOLE
}

\author{
RICHARD MARTIN \\ Université de Sherbrooke
}

En septembre 1936, les délégués du Congrès des métiers et du travail du Canada (CMTC) rassemblés à l'hôtel Mont-Royal de Montréal pour leur $52 \mathrm{e}$ réunion annuelle, votèrent une résolution d'appui aux travailleurs espagnols ainsi qu'au Front populaire:

Ce congrès désire exprimer aux travailleurs de l'Espagne son appréciation de leur lutte splendide pour la défense de leurs libertés et particulièrement de leurs institutions démocratiques et de leur gouvernement constitutionnellement élu, et affirme de nouveau au nom de la solidarité internationale l'intérêt qu'il porte aux travailleurs espagnols et leur offre son entier concours dans la lutte pour la justice, la liberté et la paix èt ses souhaits d'une victoire prochaine. ${ }^{1}$

Pour deux raisons, cette résolution peut surprendre. En effet, comment le Congrès pouvait-il concilier son anticommunisme connu avec cet appui au Front populaire, réputé communiste? Par ailleurs, cette prise de position ne laisse-t-elle pas percevoir une certaine autonomie du Congrès face à l'influence de la puissante American Federation of Labor? C'est cette dernière question qui retiendra notre attention ici.

La portée internationale du conflit espagnol et surtout son importance pour le mouvement ouvrier international sont bien connues. Voyons comment les événements ont été vécus au Canada par le Congrès des métiers et du travail. Il est d'abord important de souligner les liens qui unissaient ce syndicat à l'American Federation of Labor. Le Congrès représentait les organisations ouvrières canadiennes affiliées à des fédérations professionnelles américaines. En 1902, il s'était prononcé clairement en faveur de la liaison à

1 Gazette du travail, XXXVI (octobre 1936): 940. 
l'organisation syndicale américaine. Lors de la réunion annuelle de 1921, il définit les grands principes de sa Constitution ${ }^{2}$, et ces principes s'inspiraient largement de la philosophie du mouvement syndical américain et de son premier président, Samuel Gompers. Il n'est donc pas exagéré de lui attribuer les mêmes caractères qu'aux syndicats américains: "A labor movement upholding capitalism, not only in practice, but in principle as well.. ${ }^{3}$

Comment expliquer dès lors la prise de position du Congrès en 1936? C'est du 8 au 12 septembre 1936 que le CMTC tint sa 52e réunion annuelle, à Montréal, et c'est le jeudi 10 septembre que les délégués votèrent la résolution d'appui aux travailleurs espagnols. Selon la coutume, un représentant du syndicat britannique Trade Union Congress (TUC) ainsi qu'un représentant de l'American Federation of Labor (AFL) étaient invités aux assises annuelles du CMTC. Cependant, pour la première fois, un représentant de la Fédération internationale des syndicats ouvriers (FISO) était présent. Chacun des trois délégués, suivant la coutume, prit la parole devant les membres du CMTC. Harold Clay, du TUC, et J. Gillooly, de l'AFL, s'adressèrent à l'assistance dans la matinée du 10 septembre et, au cours de l'après-midi, ce fut au tour du représentant et secrétaire général de la FISO, Walter Schevenels.

Chose étonnante, il semble que Harold Clay ne fit pas allusion dans son allocution aux événements espagnols 4 . Or, quelques jours auparavant, les délégués du TUC, réunis à Plymouth pour leur 68e réunion annuelle, avaient évoqué la situation de l'Espagne, la jugeant menaçante. Ils avaient alors résolu d'apporter une aide financière aux ouvriers espagnols, de boycotter les biens et services allemands et d'approuver la "campagne de résistance» contre l'influence nazie et fasciste en Europe. Cette aide financière devait être supervisée par une commission mixte de la Fédération internationale des syndicats ouvriers ${ }^{5}$. Le délégué britannique ne semble pas non plus avoir fait mention de la prise de position de son propre mouvement.

Quant au représentant de l'AFL, il se limita, en matière d'affaires internationales, à attaquer la propagande subversive des

\footnotetext{
Jean-Pierre Després, Le mouvement ouvrier canadien (Montréal, 1946), 48s.

3 Selig Perlman, "The Basic Philosophy of the Labor Movement", The Annals of the American Academy (mars 1950): 61.

4 Canadian Congress Journal, XV (septembre, octobre, novembre 1936); Gazette du travail, XXXVI (février, octobre, décembre 1936).

${ }_{5}$ "British Unions favor intervening in Spain», The Toronto Daily Star, 8 septembre 1936, 3.
} 
communistes, ignorant complètement la question espagnole ${ }^{6}$. Ce mutisme pourrait s'expliquer par le fait que la réunion annuelle de l'AFL ne devait avoir lieu que deux mois plus tard, en novembre. Gillooly préférait peut-être ne pas anticiper sur la position de la centrale syndicale américaine. Pourtant, en octobre, le président de l'AFL, W. Greene, allait énoncer clairement sa position. Parlant devant les membres de l'American Legion, réunis pour leur assemblée annuelle à Cleveland, Greene déclara: «We, in United States, freely concede that the people of other nations may adopt such form of government as they may wish. ${ }^{7}$ Mais il ajouta que l'American Legion et l'AFL étaient fermement déterminées à ce que «never again shall our beloved country become involved in a European conflict. We must not become involved in a foreign war in which we have no direct concern. ${ }^{8}$ Lors du congrès annuel de l'AFL, qui s'ouvrit à Tampa, en Floride, le 16 novembre 1936, la seule résolution faisant allusion à l'Espagne qui y fut adoptée n'était qu'une "réaffirmation de l'attitude de congrès précédents relativement à l'atteinte portée à la liberté humaine et à la démocratie par le fascisme, le nazisme et le communisme" .

On est loin d'un appui franc et clair aux ouvriers espagnols, au Front populaire ou aux deux. En définitive, la position de l'AFL se résume à cette affirmation de Philip Taft: «During the Spanish Civil War, the A.F. of L. had taken no stand.»10

Par contre, l'allocution enthousiaste du représentant de la FISO devant les membres du CMTC visait deux objectifs: d'abord condamner l'action des rebelles fascistes et ensuite convaincre les membres du CMTC de favoriser le développement d'un mouvement syndical international unifié.

C'est sur un ton convaincu et convaincant que Schevenels déclare à l'assemblée que le fascisme est l'ennemi commun de la liberté, de la démocratie et du standard de vie ${ }^{11}$. D'après lui, les forces fascistes en Espagne essayaient, dans une dernière tentative, de regagner leurs pouvoirs et privilèges aux dépens de l'ensemble de la société espagnole. Aussi proclama-t-il:

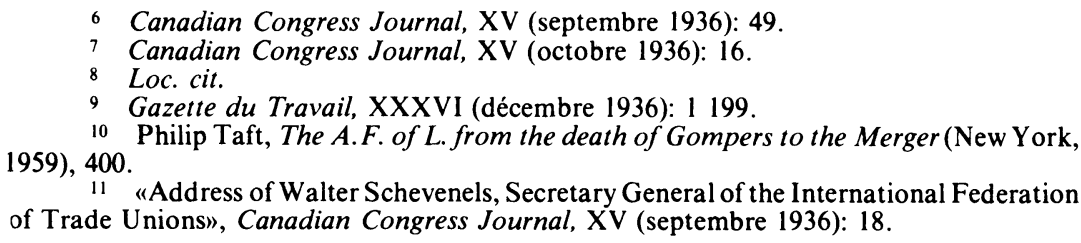


I wish to pay public tribute to the splendid spirit of self denial and incomparable bravery with which the Spanish people are defending their ideals of justice and liberty, which are also the ideals or organized labor all over the world. ${ }^{12}$

De telles paroles ont eu de toute évidence un effet déterminant sur la philosophie de la résolution votée en fin d'après-midi par les membres du CMTC, les incitant en outre à réagir dans le sens d'un geste concret, d'autant plus que Schevenels déclara:

The world is being told that the rebellion is necessary to prevent the communist and bolchevik dictatorships. I wish to say that before the reaction started in 1934, there was no communism and at the present it is ridiculous to speak of communism in Spain since that party got only fifteen seats out of 420 in the last election. ${ }^{13}$

Ainsi le discours du secrétaire général de la FISO pouvait trouver écho chez les membres du CMTC dont il respectait les grands principes idéologiques. Affirmant avec insistance que le travail, internationalement unifié, devait jouer une grande part dans le combat contre le fascisme, la guerre et la pauvreté ${ }^{14}$, Schevenels se gagna finalement l'appui du Congrès, et cet appui se concrétisa dans la résolution suivante:

Ratification de l'initiative de la Fédération des Syndicats ouvriers qui veut se rallier toutes les autres organisations ouvrières pour obtenir unité d'action et combattre la guerre et le fascisme. ${ }^{15}$

C'est en fin d'après-midi, après l'allocution de Schevenels, que fut adoptée la résolution d'appui à la lutte des ouvriers espagnols et à leur gouvernement. D'après le Globe, elle fut présentée par un torontois, John Bruce, qui dut, pour ce faire, obtenir le consentement unanime de l'assemblée puisque, selon les règles du CMTC, toutes les résolutions devaient être soumises aux délégués 20 jours avant la réunion. Cependant, comme l'écrit le Globe: «The consent was given, and, without ado, the motion was unanimously passed.,16

12 Ibid., $17 \mathrm{~s}$.

13 Ibid., 18.

14 Ibid., 17.

15 Gazette du travail (Octobre 1936): 941.

16 "T.\& L. Congress votes against Spanish rebels», The Globe, 11 septembre 1936, 3. L'article continue en disant que Gustave Franq est intervenu pour demander que la résolution soit traduite et présentée en français. Lorsque ce fut fait, les délégués adoptèrent à l'unanimité la résolution d'appui. 
Cette résolution eut un certain écho dans la presse canadienne. L'examen de quelques revues et d'une dizaine de grands journaux de Montréal, de Toronto, de Winnipeg et de Vancouver ${ }^{17}$ permet de le constater. Dans l'ensemble, l'information sur la résolution fait partie de la couverture générale de la réunion et elle ne mène ni à des prises de position éditoriales, ni à des réactions immédiates des lecteurs.

Soulignons cependant le cas particulier du Devoir, dont le rédacteur en chef, Omer Héroux, condamna violemment, dans un premier éditorial, la position du CMTC. Il lui reprochait, en somme, de se dire l'interprète du «syndicalisme neutre» alors qu'il prenait parti et qu'il se solidarisait avec ceux qui «représentent làbas l'anarchie, avec ceux que tous les éléments d'ordre en Europe considèrent comme les ouvriers, les complices ou les dupes du bolchévisme» ${ }^{18}$. Héroux visait à justifier le syndicalisme catholique et plus spécialement la Confédération des travailleurs catholiques du Canada (CTCC). Ainsi, déclara-t-il, après avoir attaqué le CMTC:

Le salut, l'ordre, les possibilités d'action féconde sont ailleurs. Aujourd'hui même la Confédération des Travailleurs Catholiques du Canada ouvre à Saint-Hyacinthe son congrès. Elle est devenue une puissance. Nous souhaitons qu'elle grandisse encore. ${ }^{19}$

Quelques jours plus tard, il dénonça la manipulation qui, selon lui, conduisait les ouvriers à appuyer inconditionnellement des causes comme celle des ouvriers espagnols ${ }^{20}$.

La revue Vie syndicale, mensuel d'allégeance catholique, réagit également en manifestant sa désapprobation à l'égard du CMTC. Elle reproduisit d'abord l'éditorial d'Omer Héroux publié dans le Devoir du samedi 12 septembre $1936^{21}$, puis dans un article relativement long, le doyen du mouvement syndical catholique, Alfred Charpentier, chercha à démontrer que l'infiltration des communistes dans les syndicats neutres, tant américains que

17 Journaux (du 8 au 19 septembre 1936): Le Canada, La Presse, The Gazette, The Montreal Star, The Globe, The Toronto Daily Star, Winnipeg Free Press, The Vancouver Sun; Le Devoir (du mois de juillet au mois d'octobre 1936). Revues (du mois de septembre au mois de novembre 1936): La vie syndicale, Canadian Forum, Canadian Unionist, Saturday Night.

18 Omer Héroux, «Unamuno, le Congrès des Métiers et du Travail et les événements d'Espagne», Le Devoir, 12 septembre 1936, 1.

19 Loc. cit.

1936.

20 Les premiers écrits dans ce sens apparaissent dans son éditorial du 14 septembre

21 Omer Héroux, "Unamuno...», La vie syndicale (septembre 1936): II. 
canadiens devenait de plus en plus forte et qu'elle était responsable à la fois de la division interne de l'AFL (qui donna naissance au CIO, en 1935) et du vote par le CMTC de la résolution en faveur du Front populaire espagnol ${ }^{22}$.

Le président du CMTC, P. M. Draper, apporta une première justification de la prise de position de son syndicat en faveur de la gauche espagnole dans l'Ottawa Journal23 et dans le Devoir ${ }^{24}$. C'est cependant dans le numéro d'octobre 1936 du Canadian Congress Journal que se trouve l'article le plus complet parce qu'il soulève certains grands principes du CMTC ${ }^{25}$. Bien qu'assez court, cet article établit très clairement que le Congrès des métiers et du travail du Canada n'est pas et n'a jamais été en faveur du communisme et que la résolution d'appui du Congrès s'inspire du grand principe qui domine l'idéologie du CMTC: une confiance inébranlable dans la forme démocratique de gouvernement et la condamnation des régimes de dictature ${ }^{26}$. En vertu de ce principe, le CMTC s'opposait donc fondamentalement au communisme, de même qu'au fascisme et au nazisme. Les délégués du CMTC auraient appuyé le Front populaire espagnol au nom de ce principe parce que le parti de la Gauche avait été démocratiquement élu en février 1936 et que l'accession de Franco au pouvoir aurait signifié l'instauration d'une dictature fasciste. Enfin, c'est aussi par solidarité syndicale que le Congrès des métiers et du travail avait voté la résolution d'appui aux ouvriers et au Front populaire espagnol.

Le rôle qu'a joué la FISO dans la décision du syndicat canadien d'appuyer les ouvriers espagnols dans leur lutte semble évident. L'influence traditionnelle de l'AFL sur le CMTC fut ainsi reléguée au second plan ce jour-là. La philosophie personnelle du président du CMTC y est sans doute pour beaucoup. P. M. Draper a toujours voulu que le syndicalisme canadien soit autonome par

22 Alfred Charpentier, "Basses intrigues des communistes», La vie syndicale (octobre 1936): I.

23 Cet article est résumé et commenté dans "The Answer to Propaganda", Canadian Congress Journal, XV (septembre 1936): 18.

24 "M. Draper et l'Espagne», Le Devoir, 24 septembre 1936, 2.

25 "Dictatorship», Canadian Congress Journal, XV (octobre 1936): 7.

26 Ce même principe fut exprimé en janvier 1936 lors de la soumission au gouvernement fédéral des revendications législatives syndicales du CMTC. Voir Gazette du travail, XXXVI (février 1936): 167. 
rapport au syndicalisme étranger. En 1902, avec quelques autres, il s'était opposé au projet de modification de la constitution du CMTC qui consacrait la dépendance du syndicalisme canadien à l'égard du syndicalisme américain ${ }^{27}$.

Cette autonomie du CMTC par rapport à l'AFL se maintiendra, tout au moins sur la question espagnole, dans les années subséquentes. En 1937, en 1938 et en 1939, les délégués du CMTC renouvelleront leur appui aux ouvriers espagnols dans leur «lutte pour leurs libertés contre le fascisme», tandis que l'AFL conservera son attitude neutre sur la question, se bornant à condamner de nouveau, dans ces années d'avant-guerre, le fascisme au même titre que le nazisme et le communisme.

L'année 1936 représente donc une date importante dans l'histoire du CMTC. Certes, l'étude de Brecher et Reisman sur les relations économiques canado-américaines, rédigée en 1957 pour la Commission Gordon, affirme que «over the past ten years there has been a discernible trend toward autonomous TLC decisions on problems of jurisdiction and recognition» ${ }^{28}$. Peut-être faudrait-il placer plus tôt les débuts de cette tendance. De toute manière, et même s'il ne faut pas exagérer l'importance de cette question, la résolution d'appui aux travailleurs espagnols nous invite à examiner cette hypothèse.

27. Charles Lipton, Histoire du syndicalisme au Canada et au Québec, 1827-1959 (Parti Pris, 1976), 207.

28 Irving Brecher et S.S. Reisman, Canada-United States Economic Relations (Ottawa, Royal Commission on Canada's Economic Prospects, juillet 1957). 214. 